\title{
Asteroid (99942) Apophis: new predictions of Earth encounters for this potentially hazardous asteroid ${ }^{\star}$
}

\author{
D. Bancelin ${ }^{1}$, F. Colas ${ }^{1}$, W. Thuillot ${ }^{1}$, D. Hestroffer ${ }^{1}$, and M. Assafin ${ }^{2,1, \star \star ~}$ \\ 1 IMCCE, Observatoire de Paris, UPMC, CNRS UMR8028, 77 Av. Denfert-Rochereau, 75014 Paris, France \\ e-mail: [david.bancelin; thuillot; colas; hestro]@imcce.fr \\ 2 Universidade Federal do Rio de Janeiro, Observatorio do Valongo, Ladeira Pedro Antonio 43, CEP 20.080 - 090 Rio De Janeiro RJ, \\ Brazil \\ e-mail: massaf@astro.ufrj.br
}

Received 30 August 2011 / Accepted 13 June 2012

\begin{abstract}
Context. The potentially hazardous asteroid (99942) Apophis, previously designated $2004 \mathrm{MN}_{4}$, is emblematic of the study of asteroids that could impact the Earth in the near future. Orbit monitoring and error propagation analysis are mandatory to predict the probability of an impact and, furthermore, its possible mitigation. Several aspects for this prediction have to be investigated, in particular the orbit adjustment and prediction updates when new astrometric data are available.

Aims. We analyze Apophis orbit and provide impact predictions based on new observational data, including several orbit propagations. Methods. New astrometric data of Apophis have been acquired at the Pic du Midi one-meter telescope (T1m) during March 2011. Indeed, this asteroid was again visible from ground-based stations after a period of several years of unfavorable conjunction with the Sun. We present here the original astrometric data and reduction, and the new orbit obtained from the adjustment to all data available at Minor Planet Center (until March 2011).

Results. We present a new sketch of keyholes and impacts for the next century. Additionally, we discuss observational errors, astrometric reduction, orbit adjustment, and adequacy of the dynamical model used.
\end{abstract}

Key words. celestial mechanics - astrometry - methods: data analysis - minor planets, asteroids: individual: Apophis

\section{Introduction}

Among the asteroids that evolve in our solar system, the near Earth asteroids (NEAs) are transient bodies that generally originate from the main belt and are transported into the inner part of the solar system through an interplay of collisions, nongravitational force drifts, and secular resonances, among other sources. The dynamical lifetime of these transient bodies is approximately of a few Myr (Morbidelli 2001). The NEOs are separated into different dynamical classes depending on their orbital parameters (semi-major axis and eccentricity). In several cases the orbit can intersect that of a terrestrial planet and lead to possible collision. These threatening orbits are primarily identified by their close distance to the Earth's trajectory (as a geometric definition, the trajectories can be close but the bodies out of phase). The MOID (minimum orbit intersection distance) can act as a warning indicator. An asteroid brighter than an absolute magnitude $H<20$ and has a MOID $\leq 0.05$ AU will be considered as potentially hazardous to the Earth (Gronchi 2005; Gronchi et al. 2007), and will need special attention to its orbit monitoring. The potentially hazardous asteroid (PHA) Apophis - previously designated $2004 \mathrm{MN}_{4}$ - is emblematic of the situation of studies of PHAs, and is one of the closest approaching asteroids to the Earth presently known. Observations gathered since its discovery in 2004 have ruled out any possibility of collision

\footnotetext{
* Based on observations made at Pic du Midi station and data from IAU-MPC.

$\star \star$ Invited researcher at IMCCE.
}

with the Earth in 2029 (Sansaturio \& Arratia 2008). However, Apophis will pass at about $33500 \mathrm{~km}$ from the Earth surface, that is, below the position of a geosynchronous orbit, and should be visible to the naked eye. Apophis will remain a companion of the Earth for decades and will show subsequent close approaches. The next one in 2036 should not be on a collisional orbit either, but could put the asteroid on an impacting trajectory for subsequent returns. The high sensitivity of the orbit to small effects, caused by the close encounter with the Earth and the gravitational pull together with the current uncertainty on the orbit and dynamical modeling, prevent any accurate prediction for the far future. On the one hand, a small change of the orbit well in advance in time can avoid any collision trajectory, on the other hand, the orbit is not sufficiently accurate to enable to predict the trajectory with high confidence. Observational data and in particular astrometric positions - are therefore mandatory to monitor the orbit of a PHA.

Here we report on new observations of (99942) Apophis made at the Pic du Midi (French Pyrénées) one-meter telescope during March 2011, i.e. during its recent apparition after a long period of conjunction with the Sun. After a brief overview in Sect. 2 of Apophis orbit and impact probabilities computed with the 2004-2008 observations, we describe in Sect. 3 the instrumentational setup and the data reduction used to obtain the astrometric position with the new observations of Apophis in March 2011. We then present the orbit adjustment and orbit propagation in Sect. 4, and discuss the results and further analysis to perform in Sect. 5 . 
Table 1. $\left(\xi_{\mathrm{N}}, \zeta_{\mathrm{N}}\right)$ coordinates and uncertainty $\left(\sigma_{\xi}, \sigma_{\zeta}\right)$ in the 2029-b plane of Apophis.

\begin{tabular}{lcc}
\hline \hline$\xi_{\mathrm{N}} \pm \sigma_{\xi}[\mathrm{km}]$ & $\zeta_{\mathrm{N}} \pm \sigma_{\zeta}[\mathrm{km}]$ & $\Delta \pm \sigma_{\Delta}[\mathrm{km}]$ \\
\hline $7126 \pm 15$ & $37406 \pm 350$ & $38080 \pm 350$ \\
\hline
\end{tabular}

Notes. The minimum distance between the center of the Earth and the asteroid is given by $\Delta$.

\section{Orbit of Apophis using 2004-2008 observations}

Asteroid Apophis may remain the most emblematic asteroid since asteroids are under surveillance. It is the only asteroid to have reached the level 4 on Torino scale for a possible impact with the Earth in 2029. Thanks to additional observations (optical and radar), the risk of 2029 has turned into a deep close approach with the Earth within $38000 \mathrm{~km}$, below the geostationary orbit area (but with an orbital inclination $\approx 40^{\circ}$ ) and with a visual magnitude $V=3.4$. Because of this deep encounter, the 2029-post orbit of Apophis is chaotic-like and consequently, some trajectories can become impact trajectories. Indeed, impacts were predicted, mainly for 2036 , but with a very low probability.

The state of the asteroid (as well as its orbit uncertainty) during the close encounter in 2029 can be studied in the b-plane (Valsecchi et al. 2003). We recall that this plane passes through the Earth's center and is perpendicular to the geocentric velocity of the asteroid. Therefore, it will have two geocentric coordinates $(\xi, \zeta)$. The projection of the ellipsoid uncertainty in this plane is just an ellipse centered on the nominal value of the coordinates $\left(\xi_{\mathrm{N}}, \zeta_{\mathrm{N}}\right)$ and with its semimajor and semiminor axis equal to $3 \sigma_{\zeta}$ and $3 \sigma_{\xi}$ respectively. Accordingly, the distance of the closest approach is equal to: $\sqrt{\xi_{\mathrm{N}}^{2}+\zeta_{\mathrm{N}}^{2}}$. Table 1 lists the nominal position $\left(\xi_{\mathrm{N}}, \zeta_{\mathrm{N}}\right)$ and uncertainty values, computed with the orbital solution $\mathrm{S}_{0}$ obtained with the optical and radar observations made during 2004-2008. It shows that the uncertainty in the asteroid position lies in the $\zeta$ component.

This gravitational pull will be such that Apophis will move from the Aten to the Apollo family and because of the possible semimajor axis values after the encounter, we expect the Earth and the asteroid to meet again after some revolutions of Apophis around the Sun and some years later. Some of those resonant returns can lead to impacts if the virtual asteroids Apophis (clones around the nominal solution) pass into a keyhole. This terminology was first used by Chodas (1999) to characterize the region in the b-plane of the first encounter in which the asteroid has to pass to collide with the Earth. Those keyholes can be primary if they are spawned by one close encounter and secondary if they are spawned by two consecutive close encounters. Because the uncertainty is along the $\zeta$ component, it is possible to represent the position of the keyholes center in the $\zeta$ distribution (Fig. 1).

Some keyholes appear to lie near the nominal solution. This vicinity does not mean that there is a high probability of an impact event. Indeed, the impact probability is directly linked to the keyhole's size, and the larger this one can be, the higher is the probability. Table 2 shows the position of the keyholes center in the $\zeta$-axis ${ }^{1}$ (LOV) and its probability (IP). The 2036-keyhole, which has a size of $\approx 600 \mathrm{~m}$ and lies far from the nominal value, has a higher impact probability than closer keyholes such as the 2076-keyhole. The value of the minimum impact distance with the Earth is also indicated.

\footnotetext{
1 The orbit uncertainty lies in the $\zeta$ component. Thus, this axis defines the Line of Variation (LOV).
}

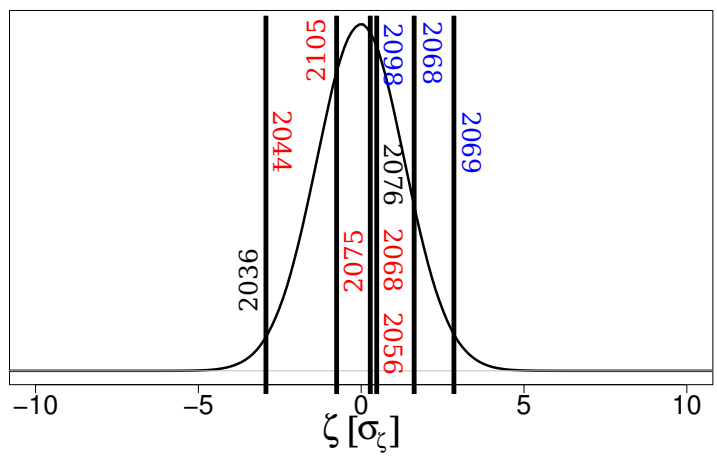

Fig. 1. $\zeta$ distribution and position of primary (black color) and secondary keyholes leading to collision at ascending node (red color) and descending node (blue color). The distribution is centered on the nominal value $\zeta_{\mathrm{N}}$ and the $\zeta$-axis is expressed in $\sigma_{\zeta}$ unit.

Table 2. Year of impact and position of the keyhole center on the LOV (in $\sigma_{\zeta}$ unit).

\begin{tabular}{cccc}
\hline \hline Year & $\begin{array}{c}\text { Distance } \\
{\left[R_{\oplus}\right]}\end{array}$ & $\begin{array}{c}\text { Position on the LOV } \\
{\left[\sigma_{\zeta}\right]}\end{array}$ & IP \\
\hline 2036 & 0.3287 & -3.394 & $2.5 \times 10^{-06}$ \\
2044 & 0.9836 & -3.346 & $2.0 \times 10^{-07}$ \\
2056 & 0.7121 & 0.308 & $2.0 \times 10^{-07}$ \\
2068 & 0.5264 & 1.051 & $2.0 \times 10^{-07}$ \\
2069 & 0.191 & 2.666 & $2.5 \times 10^{-07}$ \\
2068 & 0.1007 & 0.317 & $2.0 \times 10^{-06}$ \\
2075 & 0.3416 & 0.225 & $2.0 \times 10^{-07}$ \\
2076 & 0.2044 & 0.346 & $2.0 \times 10^{-07}$ \\
2098 & 0.7373 & 0.318 & $2.0 \times 10^{-07}$ \\
2105 & 0.1496 & -0.553 & $2.0 \times 10^{-07}$ \\
\hline
\end{tabular}

Notes. The minimum impact distance is expressed in Earth radii $\left(R_{\oplus}\right)$ and the impact probability (IP) is also indicated. The color code is the same as the one used in Fig. 1.

\section{Observations of March 2011}

Apophis was observed from 4.8 to 7.8 of March 2011 at the Pic du Midi observatory, located in the French Pyrénées (altitude $2800 \mathrm{~m}$ ). Sixty-nine observations were made with the one-meter telescope. According to the IMCCE website ${ }^{2}$, the asteroid was visible in the sky with a magnitude of 21 and the solar elongation was around $49^{\circ}$. Observations were also challenging because of its high velocity $(\sim 2.7 \mathrm{arcsec} / \mathrm{min})$. The time exposure for all observations was $90 \mathrm{~s}$. The CCD model is a "DZ936BV Marconi libandorusb". Pixel scale was 0.49 arcsec/pixel and the field of view $(\mathrm{FOV})$ is a $5.6^{\prime} \times 5.6^{\prime}$ window (the first night on March 4 was observed with a larger FOV of about $8^{\prime} \times 8^{\prime}$ with a pixel scale of $0.44 \mathrm{arcsec} / \mathrm{pixel})$. The filter was a large $R$ band, Schott RG610. A preliminary astrometry of the CCD images was made using the Astrometrica tool ${ }^{3}$. The commonly used USNO-B1.0 catalog was chosen for position reduction. Table 3 shows the prefit and posfit statistics (mean $\mu$ and standard deviation $\sigma$ ) in $(\alpha, \delta)$. The prefit statistics was computed using the residuals obtained with the astrometry performed by Astrometrica and the postfit statistics was computed using the OrbFit package ${ }^{4}$.

We have double-checked the astrometric results using an independant package, PRAIA (Assafin et al. 2010, and references

\footnotetext{
2 http://www .imcce. fr/langues/fr/

3 http://www.astrometrica.at/

4 http://adams.dm.unipi.it/orbfit/
} 
Table 3. Prefit and postfit informations, mean $\mu$ and standard deviation $\sigma$ in $(\alpha, \delta)$, for Astrometrica and the PRAIA reduction.

\begin{tabular}{lccc}
\hline \hline & & Prefit statistic & Postfit statistic \\
& & {$[\operatorname{arcsec}]$} & {$[\operatorname{arcsec}]$} \\
\hline \multirow{2}{*}{ Astr. Red. } & $\mu_{\alpha} \pm \sigma_{\alpha}$ & $-0.3629 \pm 0.4010$ & $-0.1798 \pm 0.3939$ \\
& $\mu_{\delta} \pm \sigma_{\delta}$ & $0.1244 \pm 0.2648$ & $0.1671 \pm 0.2646$ \\
\hline \multirow{2}{*}{ PRAIA Red. } & $\mu_{\alpha} \pm \sigma_{\alpha}$ & $-0.1857 \pm 0.3295$ & $-0.1129 \pm 0.3234$ \\
& $\mu_{\delta} \pm \sigma_{\delta}$ & $0.051 \pm 0.1803$ & $0.064 \pm 0.1803$ \\
\hline
\end{tabular}

therein). Object measurements were made using 2D Gaussian profiles adjusted in an iteractive procedure over central pixels within $1 F W H M$ of the center. Positions were obtained with eight 2MASS catalog stars on average, using six constant polynomial model to relate the measured and reference catalog coordinates in the tangent plane. The 2MASS reference frame was placed in the UCAC2 system by applying polynomial transformations in the tangent plane between the coordinates listed in both catalogs for common UCAC2/2MASS stars at the 2MASS epoch. The mean error of the obtained positions inferred from the $(\mathrm{O}-\mathrm{C}) \mathrm{s}$ residuals in the reference star positions were 76 mas for right ascensions and 87 mas for declinations. The dispersion of the obtained positions with regard to ephemeris is lower for the last two nights by a factor of almost two. Given the short time span and the use of the same instrument configuration and reduction procedures for the entire run, the explanation probably lies in the response of PRAIA to some change in the $\mathrm{S} / \mathrm{N}$ regime of Apophis images. Indeed, the object was severely underexposed in the images of the first two nights. For this reason, only the results of the last two nights and some results of the second night were used here. This situation is not uncommon. We are exploring the use of image co-addition centered on the moving object to improve its SNR and therefor, to improve the astrometry of past and future observations of Apophis. The PRAIA reduction enabled us to retrieve 43 observations corresponding to the last three nights. When comparing the prefit and postfit informations of the PRAIA reduction with the Astrometrica reduction (see Table 3), the mean values obtained with PRAIA reduction is obviously well below those obtained with Astrometrica.

\section{Results}

In this section, we present the results obtained thanks to the new observations from Pic du Midi and the Magadalena Ridge Observatory as available from the IAU Minor Planet Center (MPC). The fit on the observations was performed using OrbFit package. This adjustement provides the new orbital elements and the covariance matrix that will be used to estimate the accuracy on the position of Apophis during the 2029-close encounter with the Earth. The dynamical model used in the orbit propagation includes all planets and the Moon. Additionally, the perturbations from the biggest Main Belt asteroids - Ceres, Pallas and Vesta were also included. Last but not least, the relativistic corrections were also taken into account. The planetary ephemeride used is DE405. We used the Lie integrator (Bancelin et al. 2012b) where the number of terms used is 11 . This parameter represents the number of terms of the Taylor expansion for the exponential function. The internal accuracy was set to $10^{-13}$. Even if the integrator used for the orbit adjustment is not the same as the one used for the propagation (Lie integrator), Bancelin et al. (2012b) showed that the propagation of Apophis motion until the 2029-close encounter with three different integrators leads to a difference of distance less than $0.5 \mathrm{~km}$.
Table 4. 2029-b-plane uncertainties $\left(\sigma_{\xi}, \sigma_{\zeta}\right)$ and distance $\Delta_{\min }$ of Apophis from the Earth's center using four sets of observations.

\begin{tabular}{lcccc}
\hline \hline & $\mathrm{S}_{0}$ & $\mathrm{~S}_{1}$ & $\mathrm{~S}_{2}$ & $\mathrm{~S}_{3}$ \\
\hline$\sigma_{\xi}(\mathrm{km})$ & 15 & 13 & 13 & 15 \\
$\sigma_{\zeta}(\mathrm{km})$ & 350 & 217 & 217 & 245 \\
$\Delta_{\min }(\mathrm{km})$ & 38080 & 38663 & 38645 & 38350 \\
$\Delta_{\min }^{i}-\Delta_{\min }^{0}(\mathrm{~km})$ & 0 & 580 & 562 & 270 \\
\hline
\end{tabular}

Notes. The last line compares the difference of the distance with solution $\mathrm{S}_{0}$.

We compared three sets of observations, providing three new orbital solutions, with the solution $S_{0}$ obtained with the 2004-2008 data. The first one, solution $S_{1}$, was obtained using the fit of all observations available at IAU-MPC (until March 2011) including the Pic du Midi observations reduced by the USNO-B1.0 catalog. Solution $\mathrm{S}_{2}$ was obtained by using a debias treatment of all optical data available. Chesley et al. (2010) showed that biases exist in stellar catalogs (particularly in the widely used USNO-B1.0) and proposed a method to remove them from the astrometric measurements. This method has recently been implemented in the OrbFit package. To remove the biases in $(\alpha, \delta)$, we used this sofware and the files provided in the NEODyS website ${ }^{5}$. For the last three nights, the mean biases in $(\alpha, \delta)=(-120,168)$ mas are consistent with those obtained with PRAIA $(-103,170)$ mas. Finally, solution $\mathrm{S}_{3}$ was obtained using the PRAIA reduction as seen in Sect. 3.

Using a linear propagation of the covariance matrix, we gained a new estimate of the ellipse uncertainty size on the 2029-b-plane. These values show the those new optical data shrink the size of the ellipse uncertainy thanks to the orbital improvement. Accordingly, we have a better knowledge of the uncertainty on the distance of the closest approach in 2029 because it is quasi-equal to the $\sigma_{\zeta}$ value.

Solution $S_{1}$ shows that Apophis will pass $\approx 600 \mathrm{~km}$ farther from the distance computed with solution $\mathrm{S}_{0}$ and the asteroid is likely to pass close to the Earth by $38663 \mathrm{~km}$ from the Earth's center (Table 4). As a consequence, the uncertainty region on the b-plane will move up because the $\left(\xi_{N}, \zeta_{N}\right)$ coordinates have changed. As shown in Fig. 2, there is a small overlap between the dotted $3 \sigma$ ellipse $\left(\mathrm{S}_{0}\right)$ and the solid one $\left(\mathrm{S}_{1}\right)$. This is due to the size of the residuals for the first night, revealing that these observations are inconsistent. However, if the coordinates $\left(\xi_{\mathrm{N}}, \zeta_{\mathrm{N}}\right)$ computed with $\mathrm{S}_{2}$ are shifted by $(-8,-15) \mathrm{km}$ compared to $S_{1}$, solution $S_{3}$ reveals that the expected geocentric distance of Apophis seems to be less overestimated. Indeed, it seems to pass $\sim 270 \mathrm{~km}$ farther from $\mathrm{S}_{0}$, by $38350 \mathrm{~km}$. The PRAIA reduction enables us to remove the "poor observations". We will refer to solution $\mathrm{S}_{3}$ as the nominal solution.

To estimate the risk of collision, we numerically computed the position of keyholes using the Monte-Carlo technique. Another consequence of the shift of the uncertainty region is that it moves away from keyholes located below the previous solution (for instance the 2036-keyhole) and seems to come closer to keyholes located above the previous solution (for instance the 2069-keyhole). Because keyholes have a fixed position, the center of the ellipse for the nominal solution is shifted by $270 \mathrm{~km}$ from the position of the keyholes. Table 5 presents the position of the keyholes center on the LOV (in $\sigma_{\zeta}$ unit) and the impact probabilities (IP) computed with $\mathrm{S}_{3}$. The collision probability

\footnotetext{
${ }^{5}$ http://newton.dm.unipi.it/neodys/
} 


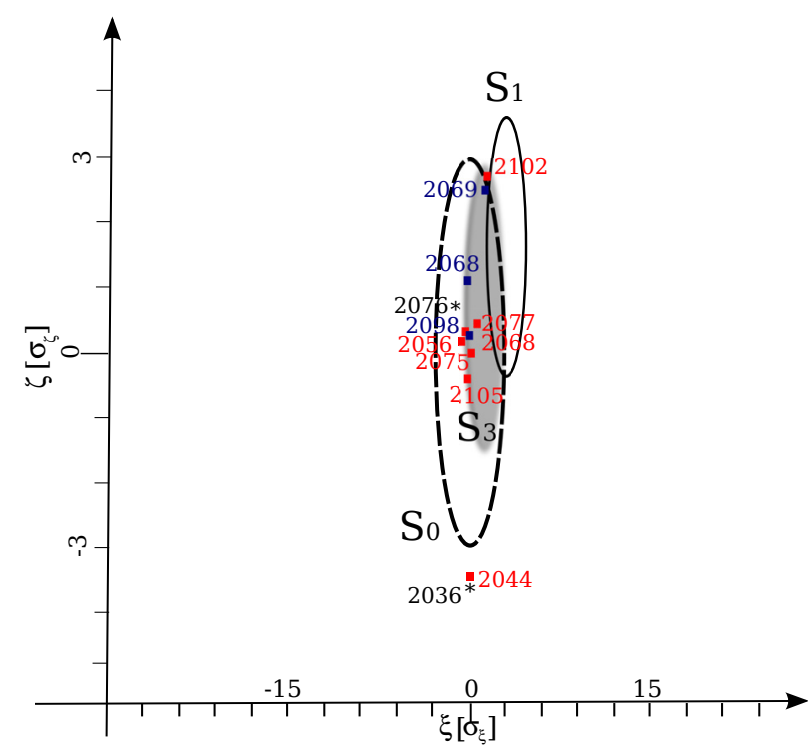

Fig. 2. 2029-b-plane of Apophis. The dotted $3 \sigma$-ellipse was computed using solution $S_{0}$. The solid one was obtained with solution $S_{1}$ and the blurred one with solution $S_{3}$. Solution $S_{2}$ is not shown because it is not significantly different from solution $S_{1}$. For completeness, the center of primary $(\star)$ and secondary keyholes leading to collision at ascending node ( $\square$ red) and descending node ( $\square$ blue) are also represented. The figure is centered on the dotted ellipse and axes are expressed in sigma units of the $\mathrm{S}_{0}$ solution with $\left(\sigma_{\xi}, \sigma_{\zeta}\right)=(15,350) \mathrm{km}$.

Table 5. Keyholes center positions on the LOV and impact probabilities of collision.

\begin{tabular}{ccccc}
\hline \hline Year & $\begin{array}{c}\text { LOV } \\
{\left[\sigma_{\zeta}\right]}\end{array}$ & IP & $\begin{array}{c}\mathrm{LOV}_{Y} \\
{\left[\sigma_{\zeta}\right]}\end{array}$ & $\mathrm{IP}_{Y}$ \\
\hline 2036 & -5.950 & $1.1 \times 10^{-07}$ & -5.862 & $4.7 \times 10^{-07}$ \\
2056 & -0.662 & $3.2 \times 10^{-07}$ & & \\
2068 & -0.649 & $2.7 \times 10^{-06}$ & -0.656 & $2.8 \times 10^{-06}$ \\
2069 & 2.705 & $3.2 \times 10^{-07}$ & 2.569 & $5.6 \times 10^{-07}$ \\
2077 & -0.795 & $2.1 \times 10^{-07}$ & -0.643 & $2.8 \times 10^{-07}$ \\
2102 & 2.767 & $2.1 \times 10^{-07}$ & & \\
\hline
\end{tabular}

Notes. $\operatorname{LOV}_{Y}$ and $\mathrm{IP}_{Y}$ refer to the position of the keyholes and impact probabilities computed using a Yarkovsky model in the orbit propagation. The color code is the same as the one used in Table 2.

is basically computed by the total fraction of clones leading to collision at the date indicated.

From the new estimate of the geocentric distance and the ellipse's uncertainty performed above we can proceed by stuying the impact of some other sources of uncertainty on these parameters. The effect of non-gravitational forces, mainly the Yarkovsky effect (Vokrouhlický et al. 2000), has already been studied by many authors (e.g. Bottke et al. 2002; Chesley 2006; Giorgini et al. 2008). The main effect of this perturbation lies in a secular drift of the semimajor axis. Because the Yarkovsky effect depends on the physical values of the asteroid, it is usually difficult to compute the accurate influence of this effect. Therefore, as suggested by Chesley (Chesley et al. 2008), it is possible to estimate the secular drift of the semimajor axis without any assumptions on the physical parameters, assuming an effect along the transverse component of this acceleration and proportionnal to $1 / r^{2}$ (with $r$ the heliocentric distance). Chesley (2006) estimated a maximum secular drift of $\pm 15 \times 10^{-4} \mathrm{AU} / \mathrm{Myr}$ as a function of the thermal conductivity and the spin orientation. The negative value is representative of the retrograde rotation and the positive value, of the prograde rotation. With this model and values, the results show that the Yarkovsky effect has a strong influence on the location of the ellipse uncertainty. Indeed, we propagated the nominal orbit from observations spanning 2004-2008, adding a transversal perturbation for the Yarkovsky effect. We found that the $(\xi, \zeta)$ coordinates are deplaced by $\pm(10,270) \mathrm{km}$ (the signs have the same meaning as for the secular drift sign). Besides, this strong influence on the location of Apophis in the 2029-b-plane can also influence the impact probability values. To this purpose, we considered this Yarkovsky model in the orbit propagation with a randomly chosen semimajor axis drift, uniformly distributed in the lower and upper bounds given above. The impact probabilities $\left(\mathrm{IP}_{Y}\right)$ and keyhole position $\left(\mathrm{LOV}_{Y}\right)$ computed with this non-gravitational effect are presented in Table 5. But, depending on the distribution used to sample the Yarkovsky displacement, these results can be significantly affected.

If there are no apparent risks of collision in the next 50 years, we can expect Apophis to have other deep close approaches. Using Monte Carlo techniques, some deep close encounters occuring in 2051 can lead to dynamical changes for Apophis on 14 April 2051: this asteroid can return to the Aten family with a low probability estimated to $2.7 \times 10^{-4}$ with an uncertainty on the date $\sim \Delta t=0.002$ days. In addition, to cause such a deflection, the asteroid will have to come close to the Earth by less than $5 R_{\oplus}$.

\section{Discussion}

Apparently, the data reduction clearly has a direct influence on the estimated location of Apophis in 2029 and hence on the impact probabilities. Owing to the difficult observational conditions, some observations of the four nights at Pic du Midi present large residuals. The PRAIA reduction (solution $\mathrm{S}_{3}$ ), corresponding to the three last nights, retrieved only the "good" observations in contrast to solution $\mathrm{S}_{1}$, for which all Pic du Midi observations were selected for orbit improvement. Since the effect of astrometric biases (solution $S_{2}$ ) were of about $20 \mathrm{~km}$ in difference of distance in 2029 and since the Yarkovsky effect should lead at most to a difference of $\pm 270 \mathrm{~km}$ in 2029 , solution $\mathrm{S}_{3}$ would seem to be more consistent with a drift from Yarkovsky force and prograde spinning body. Indeed, no strong link between the ellipse displacement and the Yarkovsky force can be deduced from this study. The Yarkovsly signal can be found by including a Yarkovsky model during the orbit adjustment process to derive physical parameters such as the spin orientation. Moreover, regarding the impact probabilities and the location of keyholes on the LOV, the Yarkovsky effect has to be taken into account during the orbit propagation to have more reliable results.

New optical data were available at MPC from late 2011 to March 2012. The minimum distance found in 2029 using those observations and the set $S_{0}$ differs by only $\approx 60 \mathrm{~km}$ compared to the distance computed with solution $\mathrm{S}_{0}$. Moreover, one can see the posfit residuals and the mean value of the residuals in Table 6, both in right ascension and declination, obtained with $S_{3}$ and the new observations of 2012. It is obvious, from the mean values of PRAIA and the new data that more importance is given to the new data and they are more consistent with the solution obtained with $\mathrm{S}_{0}$.

To proceed with this study, one can consider other resampling methods such as the Bootstrap method as applied in Desmars et al. (2009) or the Markov chain Monte Carlo method (MCMC), as applied in Oszkiewicz et al. (2009), to estimate the 
Table 6. Mean value $\mu$ and standard deviation $\sigma$ of the residuals both in right ascension and declination for PRAIA and the 2012-observations.

\begin{tabular}{lcc}
\hline \hline & & $\begin{array}{c}\text { Postfit statistic } \\
\text { [arcsec] }\end{array}$ \\
\hline \multirow{2}{*}{ PRAIA Red. } & $\mu_{\alpha} \pm \sigma_{\alpha}$ & $-0.1485 \pm 0.3232$ \\
& $\mu_{\delta} \pm \sigma_{\delta}$ & $0.054 \pm 0.1803$ \\
\hline \multirow{2}{*}{2012 Obs. } & $\mu_{\alpha} \pm \sigma_{\alpha}$ & $0.1149 \pm 0.5221$ \\
& $\mu_{\delta} \pm \sigma_{\delta}$ & $-0.0052 \pm 0.5092$ \\
\hline
\end{tabular}

Notes. They were obtained using $S_{3}$ and the new observations of 2012.

impact on the ellipse's sizes and also on the keyhole location and impact probabilities. Some dedicated observation campaigns are scheduled in 2013 and 2021 when good optical observations and radar measurements may be performed (Chesley 2006). Some space-based measurements are also expected after the launch of the satellite Gaia in early 2013. Very accurate Gaia data will significantly enhance our knowledge of Apophis' orbit (Bancelin et al. 2012a).

Acknowledgements. The authors are greatful to F. Vachier and M. Birlan for the astrometric reduction of Apophis observations, to L. Magana, undergraduate trainee at IMCCE, for her work on the observations planning and also to J.-Y. Prado from the CNES, who is at the origin of this study.

\section{References}

Assafin, M., Camargo, J. I. B., Vieira Martins, R., et al. 2010, A\&A, 515, A32

Bancelin, D., Hestroffer, D., \& Thuillot, W. 2012a, Planet. Space Sci., in press

Bancelin, D., Hestroffer, D., \& Thuillot, W. 2012b, Cel. Mech. Dyn. Astron., 4

Bottke, Jr., W. F., Vokrouhlický, D., Rubincam, D. P., \& Broz, M. 2002, The Effect of Yarkovsky Thermal Forces on the Dynamical Evolution of Asteroids and Meteoroids, 395

Chesley, S. R. 2006, in Asteroids, Comets, Meteors, eds. L. Daniela, M. Sylvio Ferraz, \& F. J. Angel, IAU Symp., 229, 215

Chesley, S. R., Vokrouhlický, D., Ostro, S. J., et al. 2008, LPI Contributions, 1405,8330

Chesley, S. R., Baer, J., \& Monet, D. G. 2010, Icarus, 210, 158

Chodas, P. W. 1999, in BAAS, 31, 1117

Desmars, J., Arlot, S., Arlot, J.-E., Lainey, V., \& Vienne, A. 2009, A\&A, 499, 321

Giorgini, J. D., Benner, L. A. M., Ostro, S. J., Nolan, M. C., \& Busch, M. W. 2008, Icarus, 193, 1

Gronchi, G. F. 2005, Cel. Mech. Dyn. Astron., 93, 295

Gronchi, G. F., Tommei, G., \& Milani, A. 2007, in IAU Symp. 236, eds. G. B. Valsecchi, D. Vokrouhlický, \& A. Milani, 3

Morbidelli, A. 2001, in Astrophys. Space Sci. Lib. 261, eds. M. Y. Marov \& H. Rickman, 289

Oszkiewicz, D., Muinonen, K., Virtanen, J., \& Granvik, M. 2009, Meteor. Planet. Sci., 44, 1897

Sansaturio, M. E., \& Arratia, O. 2008, Earth Moon and Planets, 102, 425

Valsecchi, G. B., Milani, A., Gronchi, G. F., \& Chesley, S. R. 2003, A\&A, 408, 1179

Vokrouhlický, D., Milani, A., \& Chesley, S. R. 2000, Icarus, 148, 118 